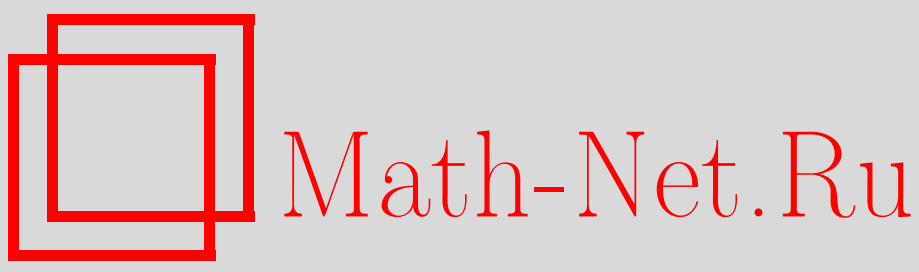

Т. П. Лукашенко, О коэффициентах систем разложения, подобных ортогональным, Матем. сб., 1997, том 188, номер 12, 57-72

DOI: https://doi.org/10.4213/sm273

Использование Общероссийского математического портала Math-Net.Ru подразумевает, что вы прочитали и согласны с пользовательским соглашением http://www . mathnet.ru/rus/agreement

Параметры загрузки:

IP: 54.174 .149 .18

26 апреля 2023 г., 16:36:13 
УДК $517.518+517.982$

\author{
Т.П. Лукашенко
}

\title{
О коэффициентах систем разложения, подобных ортогональным
}

\begin{abstract}
В работе изучаются свойства систем разложения, подобных ортогональньм. В $\S 1$ показано, что такие системы обладают большинством свойств ортогональных систем, и изучены свойства коэффициентов разложений. В $\S 2$ для ортоподобных систем разложения дан критерий, позволяющий определять, являются ли они ортогональньми проекциями ортогональных систем. В $\S 3$ показано, что для системы элементов с естественнтми условиями выполнение равенства Парсеваля эквивалентно утверждению, что это система разложения, подобная ортогональной.

Библиографиял: 11 названий.
\end{abstract}

\section{Введение}

Известно, что ортогональные системы и разложения по ним широко применяются в математике и ее приложениях, что объясняется рядом замечательных свойств таких систем. Большинство из них - равенство Парсеваля, теорема Рисса-Фишера, оценка точности приближения, известная как тождество Бесселя, сохраняются для определяемых ниже систем разложения, подобных ортогональным с неотрицательной мерой. При этом однозначности разложения, как правило, нет. В статье исследовано пространство коэффициентов разложения по таким системам, выяснена его структура; дан критерий, позволяющий определять, является ли данная система разложения ортогональной проекцией некоторой ортогональной системы; доказано, что фигурирующее в определении системы условие разложения элемента по системе при некоторых естественных ограничениях эквивалентно условию выполнения равенства Парсеваля.

\section{§1. Определения и основные свойства}

ОПРЕДЕЛЕНИЕ 1 . Пусть $H$ - гильбертово пространство над полем $\mathbb{R}$ или $\mathbb{C}$ (необязательно сепарабельное), а $\Omega$ - пространство со счетно аддитивной, соответственно, действительной или комплексной мерой $\mu$ (см. [1, гл. III, 1,2$])$. Систему элементов $\left\{e^{\omega}\right\}_{\omega \in \Omega} \subset H$ будем назьвать ортоподобной (подобной ортогональной) системой разложсения в $H$, если любой элемент $y \in H$ можно представить в виде

$$
y=\int_{\Omega} \widehat{y}_{\omega} e^{\omega} d \mu(\omega),
$$

Работа выполнена при финансовой поддержке Российского фонда фундаментальных исследований (грант № 96-01-00332), программы поддержки ведущих научных школ РФ (грант № 96/97-15-96073) и INTAS (грант № 94-1044). 
где $\widehat{y}_{\omega}=\left(y, e^{\omega}\right)$, интеграл понимается как собственный или несобственный интеграл Лебега от функции со значениями в $H$, причем в последнем случае есть такое исчерпывание $\left\{\Omega_{k}\right\}_{k=1}^{\infty}$ пространства $\Omega$ (все $\Omega_{k}$ измеримы, $\Omega_{k} \subset \Omega_{k+1}$ для $k \in \mathbb{N}$ и $\bigcup_{k=1}^{\infty} \Omega_{k}=\Omega$ ), быть может, зависяшее от $y$ и называемое подходящим для $y$, что функция $\widehat{y}_{\omega} e^{\omega}$ интегрируема по Лебегу на $\Omega_{k}$ и

$$
y=\int_{\Omega} \widehat{y}_{\omega} e^{\omega} d \mu(\omega) \equiv \lim _{k \rightarrow \infty}(L) \int_{\Omega_{k}} \widehat{y}_{\omega} e^{\omega} d \mu(\omega)
$$

ОПРЕДЕЛЕНИЕ 2. Систему элементов $\left\{e^{\omega}\right\}_{\omega \in \Omega} \subset H$ будем назьвать неотрииательной ( с неотрицательной мерой), если $\Omega$ пространство со счетно аддивной неотрицательной мерой $\mu$.

ЗАМЕЧАНИЕ 1 (об интегрировании). Используемоев определении 1 понятие интеграла является, по мнению автора, наиболее пригодным для излагаемой далее теории, хотя попытки использования других интегралов также могут быть интересны.

ЗАмечение 2 (о числовом множителе). Разумеется, в интегралы в формулах (1) и (2) можно добавить действительнозначный или комплекснозначньй множитель $a(\omega)$, но от него легко избавиться переходом к системе $\left\{s^{\omega}\right\}=\left\{\sqrt{|a(\omega)|} e^{\omega}\right\}$ и мере

$$
\eta(E)=(L) \int_{E} \frac{a(\omega)}{|a(\omega)|} d \mu(\omega)
$$

(здесь считаем $0 / 0=0$ ),

$$
(L) \int_{\Omega_{(k)}} a(\omega)\left(y, e^{\omega}\right) e^{\omega} d \mu(\omega)=(L) \int_{\Omega_{(k)}}\left(y, s^{\omega}\right) s^{\omega} d \eta(\omega),
$$

где $\Omega_{(k)}-\Omega$ или $\Omega_{k}$ (см. [1, гл. III, 10,6$\left.]\right)$. Сушествуют и другие переходы, также избавляющие от множителя $a(\omega)$.

ЗАМЕчАнИЕ 3 (об ортоподобных рядах). В случае, если $\Omega$ - конечное подмножество множества натуральных чисел $\mathbb{N}$ и для любого $k \in \Omega \mu(k)=1(>0)$, получается определение конечной неотрицательной ортоподобной системы разложения, содержащее, как частный случай, конечные полные ортонормированные (ортогональные) системы. В случае, если $\Omega=\mathbb{N}$, для любого $k \in \mathbb{N} \mu(k)=1(>0)$ и $\Omega_{k}=\{1, \ldots, k\}$ получается определение счетной неотрицательной ортоподобной системы разложения с обычной сходимостью, содержащее, как частный случай, счетные полные ортонормированные (ортогональные) системы и ряды по ним (известные как ортогональные ряды). Более чем счетные полные ортонормированные (ортогональные) системы также входят в число неотрицательных ортоподобных систем разложения.

Ортоподобные неотрицательные системы разложения по своим свойствам во многом похожи на полные ортогональные системы, как видно из нижеследующих теорем. 
ТЕорема 1 (аналог равенства Парсеваля). Если $\left\{e^{\omega}\right\}_{\omega \in \Omega} \subset H$ - неотрицательная ортоподобная система разложения в $H$, то для любого әлемента $y \in H$

$$
\|y\|^{2}=(L) \int_{\Omega}\left|\widehat{y}_{\omega}\right|^{2} d \mu(\omega)=(L) \int_{\Omega}\left|\left(y, e^{\omega}\right)\right|^{2} d \mu(\omega),
$$

для любих әлементов $y, z \in H$

$$
(y, z)=(L) \int_{\Omega} \widehat{y}_{\omega} \overline{\bar{z}_{\omega}} d \mu(\omega)=(L) \int_{\Omega}\left(y, e^{\omega}\right) \overline{\left(z, e^{\omega}\right)} d \mu(\omega)
$$

ДокАЗАТЕЛЬСтво. Так как скалярное произведение является при фиксированном втором аргументе непрерывным линейньм оператором от первого аргумента, то согласно [1, гл. III, 2, 19]

$$
\left((L) \int_{\Omega_{(k)}} \widehat{y}_{\omega} e^{\omega} d \mu(\omega), z\right)=(L) \int_{\Omega_{(k)}} \widehat{y}_{\omega} \overline{\left(z, e^{\omega}\right)} d \mu(\omega)=(L) \int_{\Omega_{(k)}} \widehat{y}_{\omega} \overline{\bar{z}_{\omega}} d \mu(\omega),
$$

где $\Omega_{(k)}$ - это или $\Omega$, или $\Omega_{k}$. В случае собственного интеграла Лебега на $\Omega$ теорема доказана. В случае несобственного интеграла, переходя к пределу при $k \rightarrow \infty$ в интегралах по $\Omega_{k}$, получаем, что

$$
\lim _{k \rightarrow \infty}(L) \int_{\Omega_{k}} \widehat{y}_{\omega} \overline{z_{\omega}} d \mu(\omega)=(y, z) .
$$

Полагая в этом равенстве $z=y$, получаем, что

$$
(L) \int_{\Omega}\left|\widehat{y}_{\omega}\right|^{2} d \mu(\omega)=\lim _{k \rightarrow \infty}(L) \int_{\Omega_{k}}\left|\widehat{y}_{\omega}\right|^{2} d \mu(\omega)=\|y\|^{2} .
$$

Так как $\left|\widehat{y}_{\omega} \overline{\widehat{z}_{\omega}}\right|$ оценивается сверху суммируемой функцией $\frac{1}{2}\left(\left|\widehat{y}_{\omega}\right|^{2}+\left|\widehat{z}_{\omega}\right|^{2}\right)$, то измеримая функция $\widehat{y}_{\omega} \overline{\bar{z}_{\omega}}$ интегрируема по Лебегу на $\Omega$ (см. [1, гл. III, 2,22$\left.]\right)$ и

$$
(L) \int_{\Omega} \widehat{y}_{\omega} \overline{z_{\omega}} d \mu(\omega)=\lim _{k \rightarrow \infty}(L) \int_{\Omega_{k}} \widehat{y}_{\omega} \overline{\bar{z}_{\omega}} d \mu(\omega)=(y, z) .
$$

Теорема доказана.

ПримечАниЕ. Можно показать, что равенство Парсеваля выполняется и в случае знакопеременной или комплексной меры при понимании интегралов в формулах (3) и (4) как несобственных интегралов Лебега.

После теоремы 1 может показаться, что естественное отображение $y \mapsto \widehat{y}_{\omega}$, $\widehat{y}_{\omega}=\left(y, e^{\omega}\right)$ является унитарным (сохраняюшим скалярное произведение) линейньм оператором изгильбертова пространства $H$ в пространство Лебега $L^{2}(\Omega)$. Но этого может не быть из-за неизмеримости функций $\widehat{y}_{\omega}=\left(y, e^{\omega}\right)-$ коэффициентов разложения. В самом деле, если $\left\{e^{\omega}\right\}_{\omega \in \Omega} \subset H$ - ортоподобная система разложения в $H$, а $\theta(\omega)$ - функция на $\Omega$ со значениями в $\mathbb{R}$ или $\mathbb{C}$ в зависимости от того, над каким полем рассматривается $H,|\theta(\omega)| \equiv 1$ на $\Omega$, то для любого элемента $y \in H$ и любого $\omega \in \Omega$ верно равенство $\left(y, \theta(\omega) e^{\omega}\right) \theta(\omega) e^{\omega}=\left(y, e^{\omega}\right) e^{\omega}$. И значит, система $\left\{\theta(\omega) e^{\omega}\right\}_{\omega \in \Omega} \subset H$ - также ортоподобная система разложения в $H$. Но функция $\left(y, \theta(\omega) e^{\omega}\right)=\overline{\theta(\omega)}\left(y, e^{\omega}\right)$ при этом может оказаться неизмеримой. В связи с этим введем следуюшее определение. 
ОПРЕДЕЛЕНИЕ 3. Неотрицательную систему $\left\{e^{\omega}\right\}_{\omega \in \Omega} \subset H$ будем называть измеримой, если все функции $\widehat{y}_{\omega}=\left(y, e^{\omega}\right)$ измеримы.

Для измеримой неотрицательной ортоподобной системы разложения отображение $y \mapsto \widehat{y}_{\omega}$ является унитарньм (сохраняющим скалярное произведение) линейньм оператором из гильбертова пространства $H$ в пространство Лебега $L^{2}(\Omega)$, и образ $H$ - замкнутое подпространство в $L^{2}(\Omega)$.

В случае счетно конечного пространства с мерой $\Omega$ любую неотрицательную ортоподобную систему можно сделать измеримой умножением на функцию, по модулю равную единице. Для доказательства этого нам понадобится следующая лемма.

ЛЕмма. Если пространство $\Omega$ со счетно аддитивной неотрицательной мерой $\mu$ счетно конечно (является не более чем счетныц обвединением множеств конечной мерь), а $\Xi$-множество функций на $\Omega$ со значениями в $\mathbb{R}$ или $\mathbb{C}$, причем для любъх двух функций $\varphi(\omega), \psi(\omega) \in \Xi$ произведение $\varphi(\omega) \overline{\psi(\omega)}$ измеримо на $\Omega$, то существует такая функция $\theta(\omega)$ на $\Omega$ со значениями, соответственно, в $\mathbb{R}$ или $\mathbb{C},|\theta(\omega)|=1$ на $\Omega$, ито для любой функции $\varphi(\omega) \in \Xi$ функиия $\overline{\theta(\omega)} \varphi(\omega)$ измерима на $\Omega$.

ДокАЗАТЕльСтво. Покажем сначала, что для любого множества конечной меры $E$ найдется такая функция $\theta(\omega)$ на $E,|\theta(\omega)|=1$ на $E$, что для любой функции $\varphi(\omega) \in \Xi$ функция $\overline{\theta(\omega)} \varphi(\omega)$ измерима на $E$.

Если все функции $\varphi(\omega)$ равны нулю почти всюду на $E$, то подходящей будет любая функция $\theta(\omega),|\theta(\omega)|=1$ на $E$, например, $\theta(\omega) \equiv 1$.

Если не все функции равны нулю почти всюду на $E$, то построим конечную или бесконечную последовательность функций $\varphi_{n}(\omega)$ следующим образом:

$$
\begin{aligned}
& \mu\left(\left\{\omega \in E|| \varphi_{n}(\omega)|>0,| \varphi_{k}(\omega) \mid=0 \text { при } 1 \leqslant k<n\right\}\right) \\
& \quad>\frac{1}{2} \sup _{\varphi \in \Xi} \mu\left(\left\{\omega \in E|| \varphi(\omega)|>0,| \varphi_{k}(\omega) \mid=0 \text { при } 1 \leqslant k<n\right\}\right),
\end{aligned}
$$

по условию функция $|\varphi(\omega)|^{2}$, а значит, и множество $\{\omega \in E|| \varphi(\omega) \mid>0\}$ измеримо для любой $\varphi \in \Xi$. Построение может оборваться только в том случае, если sup в (5) равен нулю. Теперь докажем, что на множестве

$$
E \backslash \bigcup_{n}\left\{\omega \in E|| \varphi_{n}(\omega)|>0,| \varphi_{k}(\omega) \mid=0 \text { при } 1 \leqslant k<n\right\}
$$

любая функция $\varphi(\omega)$ равна нулю почти всюду. Действительно, если бы существовала такая функция $\varphi(\omega)$, что $\mu\left\{\omega \in E|| \varphi(\omega) \mid>0, \varphi_{n}(\omega)=0\right.$ при всех $\left.n\right\}>0$, то в случае конечной последовательности это противоречило бы тому, что sup в (5) равен нулю для последнего номера $n$, а в случае бесконечной последовательности это противоречило бы тому, что в силу конечности меры $E \operatorname{sup~в~(5)~стремится~к~}$ нулю при $n \rightarrow \infty$ (ведь невозможно построить на $E$ бесконечную последовательность непересекающихся измеримых множеств, меры которых не стремятся к нулю). Определим теперь функцию $\theta(\omega)$ на $E$ следуюшим образом:

$$
\theta(\omega)= \begin{cases}\frac{\varphi_{n}(\omega)}{\left|\varphi_{n}(\omega)\right|} & \text { на }\left\{\omega \in E|| \varphi_{n}(\omega)|>0,| \varphi_{k}(\omega) \mid=0 \text { при } 1 \leqslant k<n\right\}, \\ 1 & \text { на } E \backslash \bigcup_{n}\left\{\omega \in E|| \varphi_{n}(\omega)|>0,| \varphi_{k}(\omega) \mid=0 \text { при } 1 \leqslant k<n\right\} .\end{cases}
$$


Так как по условию для любых $\varphi(\omega) \in \Xi$ и $n$ произведение $\varphi(\omega) \overline{\varphi_{n}(\omega)}$ измеримо, то построенная функция $\theta(\omega)$ на $E$ - искомая.

Пространство с мерой $\Omega$ по условию счетно конечно, его можно представить в виде не более чем счетного объединения попарно непересекающихся множеств конечной меры. Построив на каждом из них функцию $\theta(\omega)$, получим искомую функцию на всем пространстве $\Omega$.

Теорема 2 (об измеримости системы). Если $\left\{e^{\omega}\right\}_{\omega \in \Omega} \subset H$ - неотрицательная ортоподобная система разложения в $H$, а пространство с мерой $\Omega$ счетно конечно (является не более чем счетным обвединением множеств конечной меры), то существует такая функция $\theta(\omega)$ на $\Omega$ со значениями в $\mathbb{R}$ или $\mathbb{C}$ в зависимости от того, над каким полем рассматривается $H$, $|\theta(\omega)|=1$ на $\Omega$, ито $\left\{\theta(\omega) e^{\omega}\right\}_{\omega \in \Omega}-$ измеримая неотрицательная ортоподобная система разложсения в $H$.

ДокАЗАТЕЛЬСтво. Так как пространство с мерой $\Omega$ и множество функций $\Xi=\left\{\widehat{y}_{\omega}\right\}_{y \in H}$ удовлетворяют условиям леммы, функция $\theta(\omega)$ из нее и будет искомой.

В дальнейшем изложении в основном будем рассматривать измеримые неотрицательные ортоподобные системы разложения.

ОпРЕДЕЛЕНИЕ 4. Далее при рассмотрении измеримой неотрицательной ортоподобной системы разложения образ $H$ в пространстве $L^{2}(\Omega)$ при отображении $y \mapsto \widehat{y}_{\omega}$ будем обозначать $L_{H}^{2}(\Omega)$ или, короче, $L_{H}^{2}$. Это множество коэффициентов разложений элементов $y \in H$ по формуле (1):

$$
L_{H}^{2}(\Omega)=\left\{c(\omega) \in L^{2}(\Omega) \mid c(\omega)=\widehat{y}_{\omega} \text { для некоторого } y \in H\right\} .
$$

Для ортоподобных систем разложения наряду с разложениями по формуле (1) могут существовать и другие разложения. Рассмотрим их свойства.

Теорема 3. Пусть $\left\{e^{\omega}\right\}_{\omega \in \Omega}-$ измеримая неотрицательная ортоподобная система разложения в $H$, а $c(\omega)$ - функиия из пространства Лебега $L^{2}(\Omega)$ со значениями в $\mathbb{R}$ или $\mathbb{C}$ в зависимости от того, над каким полем рассматривается $H, u$

$$
y=\int_{\Omega} c(\omega) e^{\omega} d \mu(\omega)
$$

Интеграл понимается как собственный или несобственный интеграл Лебега от функиии со значениями в $H$, причем в последнем случае есть такое исчерпшвание $\left\{\Omega_{k}\right\}_{k=1}^{\infty}$ пространства $\Omega$ (все $\Omega_{k}$ измеримы,$\Omega_{k} \subset \Omega_{k+1} \partial \Omega_{\text {я }}$ $\left.k \in \mathbb{N} u \bigcup_{k=1}^{\infty} \Omega_{k}=\Omega\right)$, что функция $c(\omega) e^{\omega}$ интегрируема по Лебегу на $\Omega_{k} u$

$$
\int_{\Omega} c(\omega) e^{\omega} d \mu(\omega)=\lim _{k \rightarrow \infty}(L) \int_{\Omega_{k}} c(\omega) e^{\omega} d \mu(\omega) .
$$

Тогда для любого $z \in H$

$$
(y, z)=(L) \int_{\Omega} c(\omega) \overline{\bar{z}_{\omega}} d \mu(\omega) .
$$


ДокАЗАТЕЛЬСТво. Так как скалярное произведение является при фиксированном втором аргументе непрерывньм линейным оператором от первого аргумента, то согласно [1, гл. III, 2, 19]

$$
\left((L) \int_{\Omega_{(k)}} c(\omega) e^{\omega} d \mu(\omega), z\right)=(L) \int_{\Omega_{(k)}} c(\omega) \overline{\left(z, e^{\omega}\right)} d \mu(\omega)=(L) \int_{\Omega_{(k)}} c(\omega) \overline{\bar{z}_{\omega}} d \mu(\omega),
$$

где $\Omega_{(k)}$ - это или $\Omega$, или $\Omega_{k}$. В случае собственного интеграла Лебега на $\Omega$ теорема доказана. В случае несобственного интеграла, переходя к пределу при $k \rightarrow \infty$ в интегралах по $\Omega_{k}$, получаем, что

$$
\lim _{k \rightarrow \infty}(L) \int_{\Omega_{k}} c(\omega) \overline{\widehat{z}_{\omega}} d \mu(\omega)=(y, z)
$$

По условию $c(\omega) \in L^{2}(\Omega)$, по определению $3 \widehat{z}_{\omega} \in L^{2}(\Omega)$, значит, $c(\omega) \overline{\bar{z}_{\omega}}$ интегрируема на $\Omega$ как произведение интегрируемых в квадрате на $\Omega$ измеримых функций (см. [1, гл. III, 6,17$])$ и

$$
(L) \int_{\Omega} c(\omega) \overline{\bar{z}_{\omega}} d \mu(\omega)=\lim _{k \rightarrow \infty}(L) \int_{\Omega_{k}} c(\omega) \overline{\bar{z}_{\omega}} d \mu(\omega)=(y, z)
$$

Теорема доказана.

СлЕДСТВИЕ. При выполнении условий теоремь 3

$$
\|y\|^{2} \leqslant(L) \int_{\Omega}|c(\omega)|^{2} d \mu(\omega)
$$

причем равенство имеет место тогда и только тогда, когда $c(\omega)=\widehat{y}_{\omega}$ почти всюду на $\Omega$.

Действительно, полагая в теореме $z=y$, получаем

$$
\begin{aligned}
\|y\|^{2}=(L) \int_{\Omega} c(\omega) \overline{\hat{y}_{\omega}} d \mu(\omega) & \leqslant\left((L) \int_{\Omega}|c(\omega)|^{2} d \mu(\omega)\right)^{1 / 2} \cdot\left((L) \int_{\Omega}\left|\widehat{y}_{\omega}\right|^{2} d \mu(\omega)\right)^{1 / 2} \\
& =\left((L) \int_{\Omega}|c(\omega)|^{2} d \mu(\omega)\right)^{1 / 2} \cdot\|y\|
\end{aligned}
$$

по неравенству Коши-Буняковского-Шварца или неравенству Гёльдера для $L^{2}$ (см. $[2$, т. 2, гл. I , §5] или $[1$, гл. III, 3,2 и гл. III, 10, 42]) и по теореме 1 , причем равенство имеет место тогда и только тогда, когда $c(\omega)=\widehat{y}_{\omega}$ почти всюду на $\Omega$.

ТЕОРема 4. Пусть $\left\{e^{\omega}\right\}_{\omega \in \Omega}$ - ортоподобная неотрицательная система разложсения в $H, c(\omega)$ - функция из пространства Лебега $L^{2}(\Omega)$ со значениями в $\mathbb{R}$ или $\mathbb{C}$ в зависимости от того, над каким полем рассматривается $H$, $a\left\{\Lambda_{k}\right\}_{k=1}^{\infty}-$ такая последовательность измеримых подмнодсеств $\Omega$, что ее нижний предел (cм. [1, гл. III, 4,3]) $\underline{\lim }_{k \rightarrow \infty} \Lambda_{k}=\Omega$ и функиия $c(\omega) e^{\omega}$ интегрируема по Лебегу на $\Lambda_{k}$. Тогда в Н существует

$$
\lim _{k \rightarrow \infty}(L) \int_{\Lambda_{k}} c(\omega) e^{\omega} d \mu(\omega)
$$


ДокАЗАтельство. Действительно, $(L) \int_{\Lambda_{k}} c(\omega) e^{\omega} d \mu(\omega)$ - фундаментальная и, значит, сходящаяся последовательность, так как при $n, m>N$

$$
\begin{aligned}
& \left\|(L) \int_{\Lambda_{n}} c(\omega) e^{\omega} d \mu(\omega)-(L) \int_{\Lambda_{m}} c(\omega) e^{\omega} d \mu(\omega)\right\|^{2} \\
& \quad \leqslant(L) \int_{\Lambda_{n} \Delta \Lambda_{m}}|c(\omega)|^{2} d \mu(\omega) \leqslant(L) \int_{\Omega \backslash\left(\Lambda_{n} \cap \Lambda_{m}\right)}|c(\omega)|^{2} d \mu(\omega) \\
& \quad \leqslant(L) \int_{\Omega \backslash \cap_{k>N} \Lambda_{k}}|c(\omega)|^{2} d \mu(\omega) \underset{N \rightarrow \infty}{\longrightarrow} 0
\end{aligned}
$$

по теореме Лебега о предельном переходе под знаком интеграла [1, гл. III, 3,7$]$, ведь $|c(\omega)|^{2} \geqslant|c(\omega)|^{2} \chi_{\Omega \backslash \bigcap_{k>N} \Lambda_{k}}(\omega) \underset{N \rightarrow \infty}{\longrightarrow} 0$ всюду на $\Omega\left(\Lambda_{n} \Delta \Lambda_{m}=\left(\Lambda_{n} \cup \Lambda_{m}\right) \backslash\right.$ $\left(\Lambda_{n} \cap \Lambda_{m}\right)$ - симметрическая разность).

Доказанная теорема является аналогом известной для ортонормированных систем теоремы Рисса-Фишера (см. [3, гл. III, $\S \S 4,5],[1$, гл. IV , 16]).

СледСТвиЕ. Если $\left\{e^{\omega}\right\}_{\omega \in \Omega}$ - ортоподобная неотрииательная система разложения в $H, c(\omega)$ - функция из пространства Лебега $L^{2}(\Omega)$ со значениями в $\mathbb{R}$ или $\mathbb{C}$ в зависимости от того, над каким полем рассматривается $H$, то для любой последовательности измеримых множеств $\left\{\Omega_{k}\right\}_{k=1}^{\infty}$, нижний предел которой $\underline{\lim }_{k \rightarrow \infty} \Omega_{k}=\Omega$ (в частности, для любого исчерпьвания $\left\{\Omega_{k}\right\}_{k=1}^{\infty}$ пространства $\left.\Omega\right)$ и на әлементах $\Omega_{k}$ которой функиия $c(\omega) e^{\omega}$ интегрируема по Лебегу, существует один и тот же предел в $H$

$$
\lim _{k \rightarrow \infty}(L) \int_{\Omega_{k}} c(\omega) e^{\omega} d \mu(\omega)
$$

Действительно, из теоремы 3 следует сушествование предела для любой указанной в следствии последовательности. А то, что этот предел один и тот же для разных последовательностей, следует из того простого факта, что если $\Omega$ является нижним пределом последовательностей $\left\{\Omega_{k}\right\}_{k=1}^{\infty}$ и $\left\{\Omega_{k}^{\prime}\right\}_{k=1}^{\infty}$, то $\Omega$ является нижним пределом и смешанной последовательности $\left\{\Omega_{1}, \Omega_{1}^{\prime}, \Omega_{2}, \Omega_{2}^{\prime}, \ldots\right\}$.

ПримечАнИЕ. Вопрос о сушествовании указанных в следствии последовательностей измеримых множеств $\left\{\Omega_{k}\right\}_{k=1}^{\infty}$ не прост. Они существуют, если функция $c(\omega) e^{\omega}$ измерима как функция на $\Omega$ со значениями в $H$. Например, подходящим является исчерпьвание $\Omega$, состоящее из множеств

$$
\Omega_{k}=\left\{\omega \in \Omega\left|\frac{1}{k}<\right| c(\omega) \mid \text { и }\left\|c(\omega) e^{\omega}\right\|<k\right\} \cup\{\omega \in \Omega \mid c(\omega)=0\} .
$$

Введем определение.

ОПРЕДЕЛЕНИЕ 5. Систему $\left\{e^{\omega}\right\}_{\omega \in \Omega} \subset H$ будем называть $L^{2}$-измеримой, если для любой функции $c(\omega)$ из пространства Лебега $L^{2}(\Omega)$ со значениями в $\mathbb{R}$ или $\mathbb{C}$ в зависимости от того, над каким полем рассматривается $H$, функция $c(\omega) e^{\omega}$ измерима как функция на $\Omega$ со значениями в $H$. 
Теорема 5 (о $L^{2}$-измеримости). Если $\left\{e^{\omega}\right\}_{\omega \in \Omega}-$ неотрицательная ортоподобная система разложсения в $H$, то для ее $L^{2}$-измеримости необходимо и достаточно, чтобы выполнялись следующие условия:

а) для кажсдй функиии $c(\omega) \in L^{2}(\Omega)$ и кажсдого измеримого подмножсества $E \subset \Omega$ конечной мерьи функиия $c(\omega) e^{\omega}$ почти сепарабельнозначна на $E$;

б) для каждого $y \in H$ функиия $c(\omega) \widehat{y}_{\omega}$ измерима на $\Omega$.

Теорема непосредственно следует из [1, гл. III, 6, 11].

СледствиЕ (о $L^{2}$-измеримости). Если $\left\{e^{\omega}\right\}_{\omega \in \Omega}-$ неотрицательная ортоподобная система разложения в $H$, то для ее $L^{2}$-измеримости достаточно, чтобы она была измерима, а Н сепарабельно.

ТЕорема 6 (оценка точности приближения). Пусть $\left\{e^{\omega}\right\}_{\omega \in \Omega}-$ ортоподобная неотрицательная $L^{2}$-измеримая система разложения в $H, c(\omega)$ - функиия из пространства Лебега $L^{2}(\Omega)$ со значениями в $\mathbb{R}$ или $\mathbb{C}$ в зависимости от того, над каким полем рассматривается $H$, а $E$ - измеримое подмножество $\Omega$. Тогда существуют собственные или несобственные (как в определении 1) интегральи Лебега от функиии $c(\omega) e^{\omega}$ nо $\Omega$ и по $E$ и

$$
\left\|\int_{\Omega} c(\omega) e^{\omega} d \mu(\omega)-\int_{E} c(\omega) e^{\omega} d \mu(\omega)\right\|^{2} \leqslant \int_{\Omega \backslash E}|c(\omega)|^{2} d \mu(\omega) .
$$

ДокАЗАтЕЛЬство. Из следствия о $L^{2}$-измеримости имеем, что для любого исчерпывания $\left\{\Omega_{k}\right\}_{k=1}^{\infty}$ пространства $\Omega$, на элементах $\Omega_{k}$ которого функция $c(\omega) e^{\omega}$ интегрируема по Лебегу, сушествует один и тот же

$$
\lim _{k \rightarrow \infty}(L) \int_{\Omega_{k}} c(\omega) e^{\omega} d \mu(\omega) \equiv \int_{\Omega} c(\omega) e^{\omega} d \mu(\omega)
$$

и для любого исчерпывания $\left\{E_{k}\right\}_{k=1}^{\infty}$ множества $E$, на элементах $E_{k}$ которого функция $c(\omega) e^{\omega}$ интегрируема по Лебегу, существует один и тот же

$$
\lim _{k \rightarrow \infty}(L) \int_{E_{k}} c(\omega) e^{\omega} d \mu(\omega) \equiv \int_{E} c(\omega) e^{\omega} d \mu(\omega) .
$$

Из замечания к теореме 4 и определения 5 следует, что такие исчерпывания сушествуют. Так как

$$
\left(\int_{\Omega}-\int_{E}\right) c(\omega) e^{\omega} d \mu(\omega)=\int_{\Omega} c(\omega) \chi_{\Omega \backslash E}(\omega) e^{\omega} d \mu(\omega),
$$

а по следствию к теореме 2

$$
\begin{aligned}
& \left\|\int_{\Omega} c(\omega) \chi_{\Omega \backslash E}(\omega) e^{\omega} d \mu(\omega)\right\|^{2} \\
& \qquad(L) \int_{\Omega}\left|c(\omega) \chi_{\Omega \backslash E}(\omega)\right|^{2} d \mu(\omega)=(L) \int_{\Omega \backslash E}|c(\omega)|^{2} d \mu(\omega),
\end{aligned}
$$

то теорема 6 доказана. 
СлЕДСТвИЕ (оценка точности приближения). Пусть $\left\{e^{\omega}\right\}_{\omega \in \Omega}-$ ортоподобная неотрицательная $L^{2}$-измеримая система разложения в $H, E-$ измеримое подмножество $\Omega$. Тогда для любого әлемента $y \in H$ существует собственный или несобственный (как в определении 1) интеграл Лебега

$$
\int_{E} \widehat{y}_{\omega} e^{\omega} d \mu(\omega)
$$

$u$

$$
\left\|y-\int_{E} \widehat{y}_{\omega} e^{\omega} d \mu(\omega)\right\|^{2} \leqslant\|y\|^{2}-\int_{E}\left|\widehat{y}_{\omega}\right|^{2} d \mu(\omega) .
$$

Это утверждение непосредственно следует из теорем 4 и 1 . Оно является аналогом известной в теории рядов Фурье по ортонормированным системам оценки точности приближения - тождества Бесселя (см. [4]).

ОПРЕДЕЛЕниЕ 6. В дальнейшем при рассмотрении неотрицательной измеримой системы разложения, подобной ортогональной, будем для любого элемента $y \in H$ множество функций $c(\omega) \in L^{2}(\Omega)$, для которых вьполняется равенство (6), обозначать $L_{y}^{2}(\Omega)$ или короче $L_{y}^{2}$.

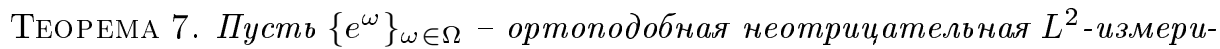
мая система разложсения в $H$, тогда подпространство $L_{0}^{2}$ - ортогональное дополнение $L_{H}^{2}$ в пространстве Лебега $L^{2}(\Omega)$. Замкнутое подпространство $L_{0}^{2}$ сдвигом на любой әлемент множества $L_{y}^{2}$ переходит в $L_{y}^{2}$. Функиия $\widehat{y}_{\omega}=\left(y, e^{\omega}\right)$ является ортогональной проекцией $L_{y}^{2}$ на $L_{H}^{2}$.

ДокАЗАТЕЛЬСТво. Из формул (1), (6), (7) и определений 3-6 сразу следует, что $L_{0}^{2}$ - ортогональное дополнение $L_{H}^{2}$ и, значит, замкнутое подпространство (см. $[1$, гл. IV , $4,3-4]$ или $[2$, т. 1 , гл. III, $\S 1])$. Так как разность любых двух элементов $L_{y}^{2}$ - элемент $L_{0}^{2}$, а сумма любого элемента $L_{y}^{2}$ с любым элементом $L_{0}^{2}$ - элемент $L_{y}^{2}$, то $L_{0}^{2}$ сдвигом на любой элемент $L_{y}^{2}$ переходит в $L_{y}^{2}$. Ортогональной проекцией $L_{y}^{2}$ на $L_{H}^{2}$ будет согласно определениям 3,4 и формуле (1) функция $\widehat{y}_{\omega}=\left(y, e^{\omega}\right)$. Теорема доказана.

В завершение параграфа отметим, что часть приведенных в нем результатов была анонсирована в [5].

\section{§2. Об ортогональных проекциях ортогональных систем}

В этом параграфе будет изложена теорема о простейшем способе построения ортоподобных неотрицательных систем разложения ортогональным проектированием, будет установлен критерий для ортоподобных неотрицательных систем разложения, которые являются ортогональными проекциями ортогональных систем, и будут приведены примеры континуальных неотрицательных систем разложения, подобных ортогональным.

Простейшим методом получения новых ортоподобных неотрицательных систем разложения является проектирование уже известных систем.

Теорема 8. Если $P$ - оператор ортогонального проектирования в гильбертовом пространстве $\mathbb{H}$ на подпространство $H, a\left\{e^{\omega}\right\}_{\omega \in \Omega} \subset \mathbb{H}-$ ортоподобная система разложения в $\mathbb{H}$ (по определению 1), то ее проекиия 
$\left\{P\left(e^{\omega}\right)\right\}_{\omega \in \Omega} \subset H$ - ортоподобная система разложения в $H$ (причем, если начальная система неотрицательна, то спроектированная система также неотрицательна, а если начальная система измерима, то спроектированная система также измерима).

ДоказАТЕльство. Если $y \in H$, то $\left(y, e^{\omega}\right)=\left(y, P\left(e^{\omega}\right)\right)$, а так как $y=P(y)$, то, используя $[1$, гл. III, 2,19$]$, получаем для случаев и собственного, и несобственного интегрирования по Лебегу, что

$$
y=P\left(\int_{\Omega}\left(y, P\left(e^{\omega}\right)\right) e^{\omega} d \mu(\omega)\right)=\int_{\Omega}\left(y, P\left(e^{\omega}\right)\right) P\left(e^{\omega}\right) d \mu(\omega) .
$$

Теорема доказана.

Эта теорема позволяет из ортонормированных и ортогональных систем с помошью проектирования получать примеры ортоподобных неотрицательных систем разложения, не являющихся ортогональными. Естественно выяснить, какие системы можно получить таким образом, а какие нельзя. Ответ содержится в следующей теореме.

ТЕОрема 9. Пусть $\left\{e^{\omega}\right\}_{\omega \in \Omega}$ - ортоподобная неотрицательная система разложсения в Н. Она является ортогональной проекиией на $Н$ некоторой полной ортонормированной (ортогональной) системь $\left\{\epsilon^{\omega}\right\}_{\omega \in \Omega}$ из какого-то содержащего $Н$ гильбертова пространства тогда и только тогда, когда для любого $\omega \in \Omega \quad \mu(\omega)=1(\mu(\omega)>0)$.

ДокАЗАтЕльство. Необходимость. Если $\left\{\epsilon^{\omega}\right\}_{\omega \in \Omega}-$ полная ортонормированная (ортогональная) система в гильбертовом пространстве $\mathbb{H} \supset H$, то для любого $y \in \mathbb{H}$ верно разложение по формуле (1) с $\mu(\omega)=1\left(\mu(\omega)=\left\|\epsilon^{\omega}\right\|^{-2}\right)$ для любого $\omega \in \Omega$ (см. [1, гл. IV $, 4,13]$ или [2, т. 2, гл. III, §4, следствие 1]). Если $\left\{e^{\omega}\right\}_{\omega \in \Omega}-$ ортогональная проекция $\left\{\epsilon^{\omega}\right\}_{\omega \in \Omega}$ на $H$, тогда $\left\{e^{\omega}\right\} \omega \in \Omega$ является ортоподобной неотрицательной системой разложения в $H$ с тем же пространством с мерой $\Omega(\mathrm{cm}$. теорему 5).

Достаточность. Если $\left\{e^{\omega}\right\}_{\omega \in \Omega}-$ ортоподобная неотрицательная система разложения в $H$ и $\mu(\omega)=1(\mu(\omega)>0)$ для любого $\omega \in \Omega$, то любое подмножество $\Omega$ измеримо, любая комплекснозначная функция $c(\omega)$ на $\Omega$ измерима, для любой функции $c(\omega)$ из пространства Лебега $L^{2}(\Omega)$ со значениями в $\mathbb{R}$ или $\mathbb{C}$ в зависимости от того, над каким полем рассматривается $H$, функция $c(\omega) e^{\omega}$ измерима (см. [1, гл. III, 6, 11]). Следовательно, $\left\{e^{\omega}\right\}_{\omega \in \Omega}-$ неотрицательная измеримая $L^{2}$-измеримая ортоподобная система разложения в $H$. Тогда характеристические функции одноточечных множеств $\chi_{\omega}$ (функции $\chi_{\omega} / \mu(\omega)$ ) образуют полную ортонормированную (ортогональную) систему в пространстве Лебега $L^{2}(\Omega)$. Так как

TO

$$
e^{\omega}=(L) \int_{\Omega} \chi_{\omega} d \mu \quad\left(e^{\omega}=(L) \int_{\Omega} \frac{\chi_{\omega}}{\mu(\omega)} d \mu\right)
$$

$$
\chi_{\omega} \in L_{e^{\omega}}^{2}\left(\frac{\chi_{\omega}}{\mu(\omega)} \in L_{e^{\omega}}^{2}\right) .
$$

В силу измеримости системы гильбертово пространство $H$ изометрически изоморфно $L_{H}^{2}$ (см. определение 4$)$, а согласно теореме 4 , следствию и примечанию к ней ортогональными проекциями функций $\chi_{\omega}(x)\left(\chi_{\omega}(x) / \mu(\omega)\right)$ на $L_{H}^{2}$ будут функции $\widehat{e}^{\omega}{ }_{x}=\left(e^{\omega}, e^{x}\right)$, соответствующие при изометрическом изоморфизме элементам $e^{\omega} \in H$. Теорема доказана. 
СлЕДСТВИЕ. Пусть $\left\{e^{\omega}\right\}_{\omega \in \Omega}-$ неотрицательная измеримая ортоподобная система разложения в $Н$. Она почти вся является ортогональной проекиией на $H$ некоторой полной ортонормированной (ортогональной) системь $\left\{\epsilon^{\omega}\right\}_{\omega \in \Omega}$ из какого-то содерэсащего $H$ гильбертова пространства тогда и только тогда, когда для почти всех $\omega \in \Omega \quad \mu(\omega)=1(\mu(\omega)>0)$.

Теперь нетрудно привести примеры ортоподобных неотрицательных систем разложения, не являющихся ортогональными проекциями ортогональных систем. Такими примерами являются континуальные системы всплесков Габора и Морле.

ПримеР 1. Порожденное одной функцией $w(x)$ семейство функций

$$
w_{\lambda, \beta}(x)=e^{2 \pi i \lambda x} w(x-\beta)
$$

где параметры $\lambda, \beta$ и переменная $x$ из $\mathbb{R}$, называют системой всплесков Габора. Если функция $w \in L^{2}(\mathbb{R})$ и $\|w\|_{L^{2}(\mathbb{R})}=1$, то для любой функции $f(x) \in L^{2}(\mathbb{R})$

$$
f(x)=\int_{\mathbb{R}} \int_{\mathbb{R}}\left(f, w_{\lambda, \beta}\right) w_{\lambda, \beta}(x) d \lambda d \beta,
$$

где последний интеграл понимается как предел в $L^{2}(\mathbb{R})$

при $A \rightarrow \infty$.

$$
\int_{\mathbb{R}} \int_{|\lambda| \leqslant A}\left(f, w_{\lambda, \beta}\right) w_{\lambda, \beta}(x) d \lambda d \beta
$$

Этот результат при дополнительном предположении суммируемости на $\mathbb{R}$ функции $w(x)$ был получен Габором (см. [6] или $[7,41.3])$. В более общем виде изложен В $[8]-[10]$.

ПРимеР 2. Порожденное одной функцией $\psi(x)$ семейство функций

$$
\psi_{\alpha, \beta}(x)=\frac{1}{\sqrt{|\alpha|}} \psi\left(\frac{x-\beta}{\alpha}\right),
$$

где параметр $\alpha \in \mathbb{R} \backslash\{0\}$, параметр $\beta \in \mathbb{R}$ и переменная $x \in \mathbb{R}$, назьвают системой всплесков Морле. Если функция $\psi \in L^{2}(\mathbb{R})$ и

$$
\int_{\mathbb{R}} \frac{|\widehat{\psi}(\lambda)|^{2}}{|\lambda|} d \lambda=1
$$

где $\widehat{\psi}(\lambda)$ - преобразование Фурье функции $\psi(x)$, то для любой функции $f(x) \in L^{2}(\mathbb{R})$

$$
f(x)=\int_{\mathbb{R}} \int_{\mathbb{R}}\left(f, \psi_{\alpha, \beta}\right) \psi_{\alpha, \beta}(x) d \beta \frac{d \alpha}{\alpha^{2}},
$$

где последний интеграл понимается как предел в $L^{2}(\mathbb{R})$

$$
\int_{|\alpha| \geqslant \varepsilon} \int_{\mathbb{R}}\left(f, \psi_{\alpha, \beta}\right) \psi_{\alpha, \beta}(x) d \beta \frac{d \alpha}{\alpha^{2}}
$$

при $\varepsilon \rightarrow+0$.

Этот результат при дополнительном предположении суммируемости на $\mathbb{R}$ функции $\psi(x)$ был получен Гроссманном и Морле (см. [11] или $[7,42.2])$. В более общем виде изложен в [8]-[10].

Заметим, что в [10] оба приведенных примера получаются при помощи некоторой общей конструкции. 


\section{§3. Критерий системы разложения - аналог равенства Парсеваля}

В этом параграфе докажем, что неотрицательная $L^{2}$-измеримая система $\left\{e^{\omega}\right\}_{\omega \in \Omega} \subset H$ будет ортоподобной неотрицательной системой разложения тогда и только тогда, когда для любого элемента $y \in H$ выполняется аналог равенства Парсеваля (3).

ЛЕмма 1. Пусть $H$ - әильбертово пространство над полем $\mathbb{R}$ или $\mathbb{C}$, $\Omega$ - пространство со счетно аддитивной действительной или комплексной мерой $\mu, a\left\{e^{\omega}\right\}_{\omega \in \Omega}-$ система әлементов из $H$. Если для каждого әлемента $y \in H$ выполняется равенство

$$
\left.\|y\|^{2}=(L) \int_{\Omega} \mid \widehat{y}_{\omega}\right)\left.\right|^{2} d \mu(\omega)
$$

где $\widehat{y}_{\omega}=\left(y, e^{\omega}\right)$, то для любых әлементов $y, z \in H$ выполняется равенство

$$
(y, z)=(L) \int_{\Omega} \widehat{y}_{\omega} \overline{z_{\omega}} d \mu(\omega)
$$

ДоКАЗАТЕЛЬСТво. Если $H$ - гильбертово пространство над полем $\mathbb{R}$, то

$$
\begin{aligned}
(y, z) & =\frac{1}{4}\left(\|y+z\|^{2}-\|y-z\|^{2}\right) \\
& =(L) \int_{\Omega} \frac{1}{4}\left(\left|\left(y+z, e^{\omega}\right)\right|^{2}-\left|\left(y-z, e^{\omega}\right)\right|^{2}\right) d \mu(\omega) \\
& =(L) \int_{\Omega}\left(y, e^{\omega}\right) \overline{\left(z, e^{\omega}\right)} d \mu(\omega)=(L) \int_{\Omega} \widehat{y}_{\omega} \overline{\bar{z}_{\omega}} d \mu(\omega) .
\end{aligned}
$$

Если $H$ - гильбертово пространство над полем $\mathbb{C}$, то

$$
\begin{aligned}
(y, z)= & \frac{1}{4}\left(\|y+z\|^{2}-\|y-z\|^{2}+i\|y+i z\|^{2}-i\|y-i z\|^{2}\right) \\
= & (L) \int_{\Omega} \frac{1}{4}\left(\left|\left(y+z, e^{\omega}\right)\right|^{2}-\left|\left(y-z, e^{\omega}\right)\right|^{2}\right. \\
& \left.\quad+i\left|\left(y+i z, e^{\omega}\right)\right|^{2}-i\left|\left(y-i z, e^{\omega}\right)\right|^{2}\right) d \mu(\omega) \\
= & (L) \int_{\Omega}\left(y, e^{\omega}\right) \overline{\left(z, e^{\omega}\right)} d \mu(\omega)=(L) \int_{\Omega} \widehat{y}_{\omega} \widehat{\bar{z}_{\omega}} d \mu(\omega) .
\end{aligned}
$$

Лемма доказана.

Лемма 2. Пусть $H$ - гильбертово пространство над полем $\mathbb{R}$ или $\mathbb{C}, \Omega$ пространство со счетно аддитивной неотрицательной мерой $\mu,\left\{e^{\omega}\right\}_{\omega \in \Omega}$ - система әлементов из $H$ и для каждого әлемента $y \in H$ выполняется равенство Парсеваля

$$
\|y\|^{2}=(L) \int_{\Omega}\left|\widehat{y}_{\omega}\right|^{2} d \mu(\omega) .
$$

Пусть некоторый әлемент $x \in H$ может быть представлен в виде

$$
x=\int_{\Omega} c(\omega) e^{\omega} d \mu(\omega),
$$


где $c(\omega)$ - функиия на $\Omega$ со значениями в $\mathbb{R}$ или $\mathbb{C}$ в зависимости от того, над каким полем рассматривается $H$, а интеграл понимается как собственныи или несобственный интеграл Лебега от функиии со значениями в $H$, причем в последнем случае есть такое исчерпьвание $\left\{\Omega_{k}\right\}_{k=1}^{\infty}$ пространства $\Omega$ (все $\Omega_{k}$ измеримьи, $\left.\Omega_{k} \subset \Omega_{k+1} \partial л я k \in \mathbb{N} u \bigcup_{k=1}^{\infty} \Omega_{k}=\Omega\right)$, что функиия $c(\omega) e^{\omega}$ интегрируема по Лебегу на $\Omega_{k}$ и

$$
\int_{\Omega} c(\omega) e^{\omega} d \mu(\omega)=\lim _{k \rightarrow \infty}(L) \int_{\Omega_{k}} c(\omega) e^{\omega} d \mu(\omega) .
$$

Tогда

$$
\|x\|^{2} \leqslant(L) \int_{\Omega}|c(\omega)|^{2} d \mu(\omega),
$$

причем равенство имеет место тогда и только тогда, когда $c(\omega)=\left(x, e^{\omega}\right)$ почти всюду на $\Omega$.

ДокАЗАТЕльство. Пользуясь непрерьвностью скалярного произведения, мы можем переставить его согласно [1, гл. III, 2, 19] с операцией интегрирования по Лебегу и с операцией предельного перехода (для несобственного интеграла Лебега). Получим

$$
(x, y)=(L) \int_{\Omega} c(\omega) \overline{\widehat{y}_{\omega}} \mu(\omega)
$$

Полагая $y=x$, имеем

$$
\begin{aligned}
\|x\|^{2} & =(L) \int_{\Omega} c(\omega) \overline{\widehat{x}_{\omega}} d \mu(\omega) \\
& \leqslant\left((L) \int_{\Omega}|c(\omega)|^{2} d \mu(\omega)\right)^{1 / 2} \cdot\left((L) \int_{\Omega}\left|\widehat{x}_{\omega}\right|^{2} d \mu(\omega)\right)^{1 / 2} \\
& =\left((L) \int_{\Omega}|c(\omega)|^{2} d \mu(\omega)\right)^{1 / 2} \cdot\|x\|
\end{aligned}
$$

по неравенству Коши-Буняковского-Шварца или неравенству Гёльдера для $L^{2}$ (см. [2, т. 2, гл. I, § 5] или [1, гл. III, 3, 2 и гл. III, 10, 42]) и по данному по условию равенству Парсеваля (при этом важна только измеримость функций $c(\omega) \widehat{\widehat{x}}_{\omega}$, $|c(\omega)|^{2}$ и $\left.\left|\widehat{x}_{\omega}\right|^{2}\right)$, причем в приведенной формуле равенство имеет место тогда и только тогда, когда $c(\omega)=\left(x, e^{\omega}\right)$ почти всюду на $\Omega$. Лемма доказана.

Лемма 3. Пусть $H$ - гильбертово пространство над полем $\mathbb{R}$ или $\mathbb{C}, \Omega$ пространство со счетно аддитивной неотрицательной мерой $\mu,\left\{e^{\omega}\right\}_{\omega \in \Omega}$ - система әлементов из $H$ и для каждого әлемента $у \in H$ выполняется равенство Парсеваля

$$
\|y\|^{2}=(L) \int_{\Omega}\left|\widehat{y}_{\omega}\right|^{2} d \mu(\omega) .
$$

Пусть $c(\omega)$ - функиия на $\Omega$ со значениями в $\mathbb{R}$ или $\mathbb{C}$ в зависимости от того, над каким полем рассматривается $H,|c(\omega)|^{2}$ интегрируема по Лебегу на $\Omega, a\left\{\Lambda_{k}\right\}_{k=1}^{\infty}$ такая последовательность измеримых подмнохсеств $\Omega$, что ее нижний предел (см. [1, гл. III, 4, 3]) $\underline{\lim }_{k \rightarrow \infty} \Lambda_{k}=\Omega$ и функция $c(\omega) e^{\omega}$ интегрируема по Лебегу на $\Lambda_{k}$. Тогда в $H$ существует

$$
\lim _{k \rightarrow \infty}(L) \int_{\Lambda_{k}} c(\omega) e^{\omega} d \mu(\omega)
$$


ДокаЗАтЕльство. Действительно, $(L) \int_{\Lambda_{k}} c(\omega) e^{\omega} d \mu(\omega)-$ фундаментальная и, значит, сходяшаяся последовательность, так как при $n, m>N$

$$
\begin{aligned}
& \left\|(L) \int_{\Lambda_{n}} c(\omega) e^{\omega} d \mu(\omega)-(L) \int_{\Lambda_{m}} c(\omega) e^{\omega} d \mu(\omega)\right\|^{2} \\
& \quad \leqslant(L) \int_{\Lambda_{n} \Delta \Lambda_{m}}|c(\omega)|^{2} d \mu(\omega) \leqslant(L) \int_{\Omega \backslash\left(\Lambda_{n} \cap \Lambda_{m}\right)}|c(\omega)|^{2} d \mu(\omega) \\
& \quad \leqslant(L) \int_{\Omega \backslash \cap_{k>N} \Lambda_{k}}|c(\omega)|^{2} d \mu(\omega) \underset{N \rightarrow \infty}{\longrightarrow} 0
\end{aligned}
$$

по предыдущей лемме и по теореме Лебега о предельном переходе под знаком интеграла [1, гл. III, 3, 7], ведь $|c(\omega)|^{2} \geqslant|c(\omega)|^{2} \chi_{\Omega \backslash \bigcup_{k>N} \Lambda_{k}}(\omega) \underset{N \rightarrow \infty}{\longrightarrow} 0$ всюду на $\Omega$ $\left(\Lambda_{n} \Delta \Lambda_{m}=\left(\Lambda_{n} \cup \Lambda_{m}\right) \backslash\left(\Lambda_{n} \cap \Lambda_{m}\right)\right.$ - симметрическая разность). Лемма доказана.

СЛЕДСТВИЕ. Пусть $H$ - гильбертово пространство над полем $\mathbb{R}$ или $\mathbb{C}, \Omega$ - пространство со счетно аддитивной неотрицательной мерой $\mu,\left\{e^{\omega}\right\} \omega \in \Omega$ - система әлементов из $H$ и для каждого әлемента $у \in H$ выполняется равенство Парсеваля

$$
\|y\|^{2}=(L) \int_{\Omega}\left|\widehat{y}_{\omega}\right|^{2} d \mu(\omega) .
$$

Если $c(\omega)$ - функиия на $\Omega$ со значениями в $\mathbb{R}$ или $\mathbb{C}$ в зависимости от того, над каким полем рассматривается $H,|c(\omega)|^{2}$ интегрируема по Лебегу на $\Omega$, то для любой последовательности измеримых множсеств $\left\{\Omega_{k}\right\}_{k=1}^{\infty}$, нижний предел которой $\underline{\lim }_{k \rightarrow \infty} \Omega_{k}=\Omega$ (в частности, для любого исчерпьвания $\left\{\Omega_{k}\right\}_{k=1}^{\infty}$ пространства $\left.\Omega\right)$ ина әлементах $\Omega_{k}$ которой функиия $c(\omega) e^{\omega}$ интегрируема по Лебегу, существует один и тот же предел в $H$

$$
\lim _{k \rightarrow \infty}(L) \int_{\Omega_{k}} c(\omega) e^{\omega} d \mu(\omega)
$$

Действительно, из леммы 3 следует сушествование предела для любой указанной в следствии последовательности. А то, что этот предел один и тот же для разных последовательностей, следует из того простого факта, что если $\Omega$ является нижним пределом последовательностей $\left\{\Omega_{k}\right\}_{k=1}^{\infty}$ и $\left\{\Omega_{k}^{\prime}\right\}_{k=1}^{\infty}$, то $\Omega$ является нижним пределом и смешанной последовательности $\left\{\Omega_{1}, \Omega_{1}^{\prime}, \Omega_{2}, \Omega_{2}^{\prime}, \ldots\right\}$.

ПримечАниЕ. Множество указанных в следствии последовательностей не пусто, если функция $c(\omega) e^{\omega}$ измерима как функция на $\Omega$ со значениями в $H$. Например, подходящим является исчерпьвание $\Omega$, состоящее из множеств

$$
\Omega_{k}=\left\{\omega \in \Omega\left|\frac{1}{k}<\right| c(\omega) \mid \text { и }\left\|c(\omega) e^{\omega}\right\|<k\right\} \cup\{\omega \in \Omega \mid c(\omega)=0\} .
$$

ТеОРема 10. Пусть $H$ - гильбертово пространство над полем $\mathbb{R}$ или $\mathbb{C}, \Omega$ - пространство со счетно аддитивной неотрицательной мерой $\mu,\left\{e^{\omega}\right\} \omega \in \Omega$ - $L^{2}$-измеримая система әлементов из $H$ и для каждого әлемента $у \in H$ выполняется равенство Парсеваля

$$
\|y\|^{2}=(L) \int_{\Omega}\left|\widehat{y}_{\omega}\right|^{2} d \mu(\omega) .
$$

Тогда $\left\{e^{\omega}\right\}_{\omega \in \Omega}$-ортоподобная $L^{2}$-измеримая неотрицательная система разложения в $H$. 
ДокАЗАТЕЛЬСтво. Для любого $y \in H$ по следствию к лемме 3 сушествует собственный или несобственный интеграл Лебега

$$
I=\int_{\Omega} \widehat{y}_{\omega} e^{\omega} d \mu(\omega)
$$

Рассмотрим $\|I-y\|^{2}=\|I\|^{2}+\|y\|^{2}-(I, y)-\overline{(I, y)}$. По лемме 2

$$
\|I\|^{2} \leqslant(L) \int_{\Omega}\left|\widehat{y}_{\omega}\right|^{2} d \mu(\omega) .
$$

По условию теоремы

$$
\|y\|^{2}=(L) \int_{\Omega}\left|\widehat{y}_{\omega}\right|^{2} d \mu(\omega) .
$$

По лемме 1

$$
\left.(I, y)=(L) \int_{\Omega} \widehat{y}_{\omega} \overline{\hat{y}}_{\omega} d \mu(\omega)=(L) \int_{\Omega} \mid \widehat{y}_{\omega}\right)\left.\right|^{2} d \mu(\omega) .
$$

Из этой формулы видно, что $(I, y)$ - действительное число и, следовательно, $\overline{(I, y)}=(I, y)$. Из выписанных формул получаем, что

$$
\|I-y\|^{2}=\|I\|^{2}+\|y\|^{2}-(I, y)-\overline{(I, y)} \leqslant 0 .
$$

Теорема доказана.

СлЕДСТвиЕ (критерий неотрицательной $L^{2}$-измеримой ортоподобной системы разложения). Пусть $H$ - гильбертово пространство над полем $\mathbb{R}$ или $\mathbb{C}, \Omega$ пространство со счетно аддитивной неотрицательной мерой $\mu,\left\{e^{\omega}\right\}_{\omega \in \Omega}-$ $L^{2}$-измеримая система әлементов из $H$. Каждый әлемент у $\in H$ может быть представлен в виде

$$
y=\int_{\Omega} \widehat{y}_{\omega} e^{\omega} d \mu(\omega)
$$

где интеграл понимается как собственный или несобственный интеграл Лебега от функиии со значениями в $H$, тогда и только тогда, когда для каждого әлемента $y \in H$ выполняется равенство Парсеваля

$$
\|y\|^{2}=(L) \int_{\Omega}\left|\widehat{y}_{\omega}\right|^{2} d \mu(\omega) .
$$

Tеорема 11. Пусть $H$ - сепарабельное гильбертово пространство над полем $\mathbb{R}$ или $\mathbb{C}$, пространство $\Omega$ со счетно аддитивной неотрицательной мерой $\mu$ счетно конечно $\left\{e^{\omega}\right\}_{\omega \in \Omega}-$ система әлементов из $H$ и для каждого элемента $y \in H$ выполняется равенство Парсеваля

$$
\|y\|^{2}=(L) \int_{\Omega}\left|\widehat{y}_{\omega}\right|^{2} d \mu(\omega) .
$$

Тогда $\left\{e^{\omega}\right\}_{\omega \in \Omega}-$ неотрицательная ортоподобная система разложения в $H$. 
ДокАЗАТЕЛЬСтво. Так как пространство с мерой $\Omega$ и множество функций $\Xi=\left\{\widehat{y}_{\omega}\right\}_{y \in H}$ удовлетворяют условиям леммы, то сушествует такая функция $\theta(\omega)$ на $\Omega$, что для любого $y \in H$ функция $\overline{\theta(\omega)} \widehat{y}_{\omega}$ измерима на $\Omega$. Система $\left\{\theta(\omega) e^{\omega}\right\}_{\omega \in \Omega}$ - неотрицательная измеримая система в сепарабельном гильбертовом пространстве $H$, по теореме 5 она $L^{2}$-измерима. Тогда по теореме $10 \quad\left\{\theta(\omega) e^{\omega}\right\}_{\omega \in \Omega}-$ ортоподобная $L^{2}$-измеримая неотрицательная система разложения в $H$, а значит, и $\left\{e^{\omega}\right\}_{\omega \in \Omega}$ - ортоподобная $L^{2}$-измеримая неотрицательная система разложения в $H$. Теорема доказана.

Для приведенных ранее систем Габера и Морле доказательство выполнения равенства Парсеваля (3) сушественно проше доказательства выполнения формулы представления (1) (см. [7, 41.3, 42.2] или [6], [11]). Подобная ситуация имеет место и для ряда других систем.

В заключение отметим, что приведенные результаты свидетельствуют, по мнению автора, что системы разложения, подобные ортогональным, являются интересньм для дальнейшего изучения объектом.

\section{Список литературы}

1. Данфорд Н., Швари Дж. Т. Линейные операторы. Обшая теория. Т. 1. М.: ИЛ, 1962.

2. Иосида К. Функциональньй анализ. М.: Мир, 1987.

3. Колмогоров А. Н., Фомин С. В. Элементы теории функций и функционального анализа. М.: Наука, 1989.

4. Бесселя неравенство // Математическая энциклопедия. Т. 1. М.: Советская энциклопедия, 1977. С. 459.

5. Лукашенко Т. П. О системах разложения, подобных ортогональнтм // Международная конф. по теор. приближ. функц., посв. памяти проф. П. П. Коровкина. Калуга, 26-29 июня 1996 г. Тезисы докл. Т. 2. Тверь: Изд-во ТГУ, 1996. С. 135-136.

6. Gabor D. Theory of communication // J. Inst. Elec. Eng. (London). 1946. V. 93 (3). P. $429-457$.

7. Gasquet C., Witomski P. Analyse de Fourier et applications. Paris: Masson, 1990.

8. Лукашенко Т. П. Всплески на топологических группах // Докл. АН. 1993. Т. 332. № 1. C. $15-17$.

9. Лукашенко Т. П. Всплески на топологических группах // Изв. РАН. Сер. матем. 1994. T. 58. №3. C. $88-102$.

10. Лукашенко Т. П. Системы разложения на пространствах с мерой // Изв. РАН. Сер. матем. 1996. Т. 60. № 1. С. 165-174.

11. Grossmann A., Morlet J. Decomposition of Hardy functions into square integrable wavelets of constant shape // SIAM J. Math. Anal. 1984. V. 15. P. 723-736. 